\title{
ESTUDO DA VIABILIDADE DE MÉTODOS GEOESTATÍSTICOS NA MENSURAÇÃO DA VARIABILIDADE ESPACIAL DA DUREZA DA MADEIRA DE PARAJU (Manilkara sp) ${ }^{1}$
}

Julião Soares de Souza Lima ${ }^{2}$, José Tarcisio Oliveira da Silva², Rone Batista de Oliveira ${ }^{3}$, Vinícius Silva de Almeida ${ }^{4}$ e Felipe Lyra Vanzo ${ }^{4}$

\begin{abstract}
RESUMO - Este trabalho objetivou determinar a viabilidade do uso da metodologia geoestatística na predição do padrão espacial da dureza, como propriedade mecânica da madeira de paraju (Manilkara sp). A peça de madeira apresentou as dimensões de 25,0 x 7,0 x 75,0 cm, correspondente a largura, espessura e comprimento, respectivamente. As duas faces da peça foram divididas em malhas regulares ("grid") de 2,5 x 2,5 cm composta de 9 linhas e 29 colunas, totalizando 261 compartimentos denominados células. A determinação da dureza da madeira foi realizada com base no método de Janka, com a carga aplicada no centro de cada célula. Os dados foram submetidos à análise estatística descritiva, geoestatística e interpolação por "krigagem”. Os valores do coeficiente de variação apresentaram-se baixos. Observou-se uma dependência espacial para a dureza da madeira em ambas as faces, com maior alcance ocorrendo na face A da peça. Conclui-se que essa técnica tem aplicação prática no fornecimento de subsídios para retirada de amostras em peças de madeira, havendo, no entanto, necessidade de outros estudos.
\end{abstract}

Palavras-chave: Propriedades mecânicas, amostragem e interpolação.

\section{VIABILITY STUDY OF GEOSTATISTICAL METHODS IN THE SPATIAL VARIABILITY MENSURATION OF PARAJU WOOD HARDNESS (Manilkara sp)}

\begin{abstract}
This work aimed at analyzing the feasibility of geostatic methodology in estimating the spatial pattern of paraju (Manilkara sp) wood hardness. The studied timber piece dimensions were $25 \times 7 \times 75 \mathrm{~cm}$ in width, thickness and length respectively. The two sides of the piece were divided in regular $2.5 \times 2.5 \mathrm{~cm}$ grids comprising nine rows and twenty nine columns, totaling two hundred sixty one cells. The hardness essay was based on the Janka method with the force applied in the middle of each cell. Data were examined by descriptive statistical analyses, geostatistic and krigage interpolation. The variation coefficients were low. Spatial for wood hardness dependence was found in both sides, with higher values in side A of the piece. It was concluded that this method has practical use for providing information on sampling of timber pieces, however, there is need for further studies.
\end{abstract}

Keywords: Mechanics properties, sampling and interpolation.

\footnotetext{
${ }^{1}$ Recebido em 10.02.2005 e aceito para publicação em 05.04.2006.

${ }^{2}$ Departamento de Engenharia Rural da Universidade Federal do Espírito Santo, Alegre-ES. E-mail: <juliaosslima@ cca.ufes.br

${ }^{3}$ Programa de Pós-Graduação em Produçaõ Vegetal da UFES.

${ }^{4}$ Engenheiro Florestal.
} 


\section{INTRODUÇÃO}

A teoria das variáveis regionalizadas, desenvolvida por Krige (1951), conforme citado por Vieira (2000), concluiu que somente a informação dada pela variância dos dados é insuficiente para explicar um fenômeno em estudo. Para tal, seria necessário levar em consideração a distância entre as observações. A partir daí surgiu o conceito de geoestatística. Essa teoria passou a ser utilizada em diversos campos do conhecimento, como hidrologia, ciência do solo, ciência florestal e estudos de poluição, entre outros, algumas dessas áreas viram essa técnica como uma ferramenta adequada para análise dos dados.

É importante assinalar que a geoestatística não se refere a um tipo especial, diferente ou alternativo de estatística. O fato é que cada observação é descrita não apenas pelo seu valor, mas também das informações de sua posição, expressa por um sistema de coordenadas. O valor da variável em uma localidade pode ser, ao menos parcialmente, predito pelos valores em pontos vizinhos que não são estocasticamente independentes. Portanto, essa teoria considera a dependência espacial das observações, lançando mão de medidas estatísticas que expressem essa estrutura de dependência em pontos referenciados (RIBEIRO JUNÍOR, 1995).

O estudo das variáveis regionalizadas está sendo utilizado, com mais freqüência, em trabalhos na área de ciência do solo. Inicialmente, o enfoque dessa teoria foi voltado para problemas de mineração; subseqüentemente, sua aplicação se estendeu para outros ramos, inclusive para a ecologia, de forma a utilizar métodos para descrever o padrão de distribuição dos organismos no espaço (ROBERTSON, 1987). Na área da ciência florestal, essa técnica foi utilizada mais precisamente no manejo florestal desenvolvido por Ushizima et al. (2003), na detecção e predição do padrão espacial da espécie Anadenanthera peregrina (angicovermelho). Concluiu-se que esse método caracteriza espacialmente a distribuição, diferenciando localidades com maior agregação de indivíduos das áreas com menor agregação e, ainda, as áreas desprovidas de indivíduos, bem como subsídios para exploração racional de recursos florestais, de maneira a otimizar a relação entre volume de madeira obtida e tamanho da área.

Na ciência florestal, mais precisamente na área de tecnologia da madeira não é comum encontrar trabalhos na literatura considerando a variabilidade espacial de propriedades físicas e mecânicas da madeira ou sua correlação com a posição de amostragem (coordenadas) em determinada peça. Sabe-se da existência de grande variabilidade nas propriedades da madeira dentro de um mesmo tronco, sendo esta muito elevada para determinadas espécies lenhosas, sobretudo na direção radial ou no sentido medula-casca. Tal variabilidade nas propriedades da madeira é objeto de muita investigação, em que na madeira de eucalipto se pode citar trabalhos de Oliveira (1998); Silva (2002) e Alzate (2004), dentre muitos outros.

O conhecimento de determinada característica em locais não amostrados pode ser feita pelo método da "krigagem", técnica usada na geoestatística com os pesos atribuídos de acordo com a variabilidade espacial expressa no semivariograma (VIEIRA et al., 1998), com base nos seus vizinhos. A "krigagem" se comporta como um interpolador ótimo, pela maneira como os pesos são distribuídos, não sendo tendenciosos, apresentando variância mínima e possibilitando o conhecimento da variância da estimativa (WEBSTER e OLIVER, 1980).

Este estudo teve como objetivo determinar a viabilidade do uso da metodologia geoestatística na predição do padrão espacial da dureza como propriedade mecânica da madeira de paraju (Manilkara sp).

\section{MATERIAL E MÉTODOS}

O ensaio de dureza foi realizado no Laboratório de Ciência da Madeira do Núcleo de Estudos e de Difusão de Tecnologia em Florestas, Recursos Hídricos e em Agricultura Sustentável-NEDTEC, do Centro de Ciências Agrárias da Universidade Federal do Espírito Santo UFES, situado no Município de Jerônimo Monteiro, ES.

Utilizou-se uma peça de paraju (Manilkara sp), proveniente do comércio da região, a ser utilizada na construção e estrutura de telhado. A peça apresentou as dimensões de 25,0 x 7,0 x 75,0 cm correspondentes à largura, espessura e comprimento, respectivamente. Os teores de umidade da peça nas faces A e B foram de 21,85 e $22,08 \%$, e as massas específicas aparentes ao ar foram de $1,14 \mathrm{e} 1,15 \mathrm{~g} / \mathrm{cm}^{3}$, respectivamente. Propriedades essas determinadas segundo MB-26 (1940), da ABNT.

Primeiramente, as duas faces da peça foram divididas em malhas regulares ("grid") de 2,5 x 2,5 cm, composta de nove linhas e 29 colunas, totalizando 261 compartimentos, denominados células. As cargas foram aplicadas no centro de cada célula. 
A Figura 1 ilustra a distribuição dos anéis de crescimento na seção transversal da peça de madeira estudada, bem como o sentido de aplicação das cargas nas duas faces.

A determinação da dureza da madeira foi realizada segundo o método de Janka, preconizado na norma MB-26/40, da ABNT (1940), que consiste em medir o esforço necessário para introduzir na madeira uma semiesfera de aço de $1 \mathrm{~cm}^{2}$ de seção diametral até uma profundidade igual ao seu raio no tempo de carga em torno de $1 \mathrm{~min}$. O ensaio foi realizado em uma máquina universal de ensaio, de marca EMIC, modelo DL-10.000, totalmente automatizada.

Para analisar os dados, realizou-se, primeiramente, uma análise exploratória, com o intuito de descrever os parâmetros estatísticos que ajudam a identificar a tendência, dispersão e forma de distribuição dos dados. A hipótese de normalidade dos dados foi testada pelo teste de Lilliefors, usando-se o software SAEG.

Na etapa da análise da variabilidade espacial, os dados foram analisados, utilizando-se técnicas de geoestatística, com o intuito de identificar e definir a estrutura de dependência espacial dos dados, com base nas pressuposições de estacionaridade da hipótese intrínseca, pelo gráfico "post plot” $e$ pela análise de semivariogramas, ajustados pelo software GS+ (ROBERTSON, 1998), bem como o grau de dependência espacial entre os pontos de ensaio. A análise de dependência espacial é baseada na suposição de que medições separadas por distâncias pequenas são mais semelhantes umas às outras que aquelas separadas por distâncias maiores. Os semivariogramas foram estimados pela seguinte expressão:

$$
\gamma(h)=\frac{1}{2 N(h)} \sum_{i=1}^{N(h)}[Z(x i)-Z(x i+h)]^{2}
$$

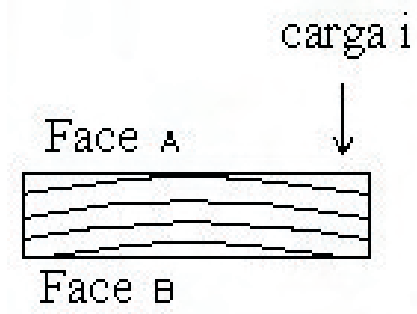

Figura 1 - Esquema representativo da seção transversal da peça de madeira de paraju.

Figure 1 - Cross section of paraju wood piece.
$\mathrm{N}(\mathrm{h})$ é o número de pares experimentais de dados separados por uma distância $\mathrm{h}, \mathrm{Z}$ (xi)é o valor determinado em cada ponto de leitura e $\mathrm{Z}(\mathrm{xi}+\mathrm{h})$ é o valor medido num ponto, mais uma distância $h$.

Testaram-se semivariagramas do tipo esférico, exponencial, linear, linear com patamar e gaussiano (SOUZA et al., 2004). A escolha dos modelos matemáticos foi realizada, observando-se o coeficiente de correlação obtido pela técnica de validação cruzada. Essa técnica consiste em retirar, individualmente, cada ponto medido da área estudada; o seu valor é estimado, via "krigagem", como se ele nunca existisse.

Para a determinação e visualização do comportamento da variável na região, por meio de um mapa de isolinhas, em locais não medidos, utilizouse o método da "krigagem" ordinária, que consiste num estimador linear não-viciado com mínima variância para a interpolação da variável medida em posições não-amostradas (ISAAKS e SRIVASTARA, 1989).

\section{RESULTADOS E DISCUSSÃO}

Os resultados referentes à análise descritiva da dureza da madeira estão apresentados no Quadro 1.

A distribuição dos dados apresenta assimetria negativa para as duas faces da peça; nesse caso, a média é menor que a moda e a mediana, conforme mostrado no Quadro 1. O valor do coeficiente de curtose para o lado A apresenta distribuição planicurdica e o lado B, distribuição leptocurdica. O software (GS+) utilizado adota o valor zero para distribuição mesocurdica.

Quadro1 - Análise estatística descritiva da dureza $\left(\mathrm{kgf} / \mathrm{cm}^{2}\right)$ Table 1 -Descriptive statistical analysis of hardness $\left(\mathrm{kgf} / \mathrm{cm}^{2}\right)$

\begin{tabular}{lcc}
\hline Estatística & \multicolumn{2}{c}{ Variáveis Mecânicas } \\
\cline { 2 - 3 } & Dureza (Face A) & Dureza (Face B) \\
\hline No de obsevações $_{\text {Média }}$ & 261 & 261 \\
Moda & 1109 & 1000 \\
Mínimo & 1122 & 1033 \\
$1^{\circ}$ quartil & 904 & 657 \\
Mediana & 1056 & 943 \\
$3^{\circ}$ quartil & 1119 & 1012 \\
Máximo & 1162 & 1061 \\
Desvio-Padrão & 1270 & 1166 \\
Variância & 71 & 84 \\
CV(\%) & 5003 & 7064 \\
Assimetria & 6,38 & 8,54 \\
Curtose & $-0,30$ & $-0,58$ \\
P $^{*}$ - valor & $-0,26$ & 0,59 \\
\hline
\end{tabular}

Valor de $\mathrm{P}<0,05$ para o teste de Normalidade de Lilliefors 
Os coeficientes de variação (CV) são de $6,38 \%$ na face A e $8,54 \%$ na face B. Segundo Pimentel Gomes (1987), dados com CV inferiores a $10 \%$ podem ser considerados como de baixa variabilidade.

Os resultados referentes ao teste Lilliefors indicaram normalidade para a variável dureza nas duas faces estudadas. Segundo Isaaks e Srivastava (1989), mais importante que a normalidade dos dados é a ocorrência ou não do chamado efeito proporcional, em que a média e a variabilidade dos dados sejam constantes no estudo; no entanto, em se tratando de dados obtidos na natureza, o ajuste de uma distribuição teórica é apenas aproximado (WARRICK e NIELSEN, 1980).

As médias de dureza nas duas condições são distintas: na face Aé de $1109 \mathrm{kgf} / \mathrm{cm}^{2}$ e na face B, 1000 $\mathrm{kgf} / \mathrm{cm}^{2}$. A diferença apresentada entre as faces dessa variável é de 10,94\%, sendo maior na face A. Essa diferença se deve à maior idade cambial da madeira formada na face $\mathrm{A}$, o que proporcionou a formação de tecido lenhoso de maior densidade e, conseqüentemente, maior dureza.

As análises de estacionaridade ou tendência direcional da variável em estudo estão nos gráficos "post plot", mostrados nas Figuras 2 e 3, nas faces A e B, respectivamente. Desse modo, os intervalos, em que aparecem concentrações de cores iguais, indicam a existência de uma sub-região no mapa, em função dos quartis. Observa-se, na Figura 2, que a dureza apresentou baixos valores na parte inferior e maiores valores na parte superior. Esses dados indicaram uma leve descontinuidade na direção Y (colunas) e maior normalidade na direção do comprimento X (linhas). A face B da peça, mostrada na Figura 3, apresentou um comportamento sem tendência na distribuição da dureza. Pode-se notar que, comparando a variação da dureza em relação às duas faces da peça, observa-se que, na sub-região dos menores valores na face $\mathrm{A}$, há correspondência a uma concentração da sub-região de maior dureza, na face $B$.

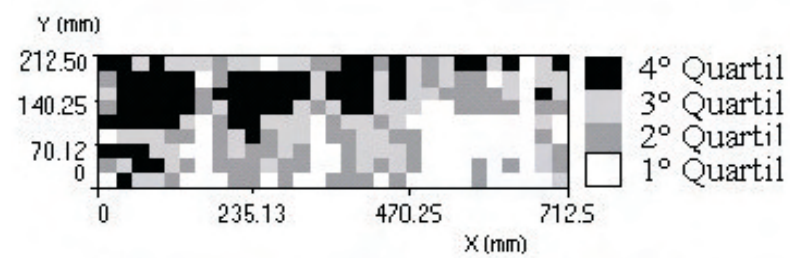

Figura 2 - Gráfico Post plot da variável dureza na peça de paraju na face A e sua divisão em quartil.

Figure 2 - Post plot graph of variable hardness in paraju piece side $A$ and its quartile division.

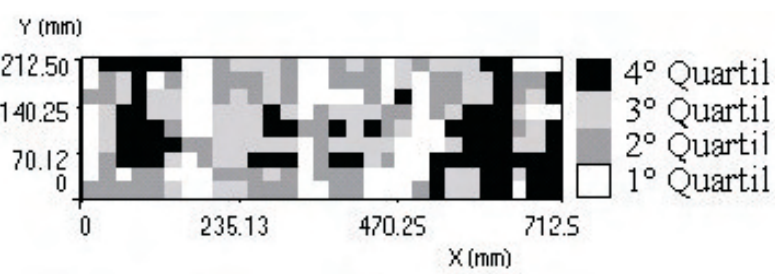

Figura 3 - Gráfico Post plot da variável dureza na peça de paraju na face B e sua divisão em quartil.

Figure 3 - Post plot graph of variable hardness in paraju piece side $B$ and its quartile division.

Outra forma de identificar a estacionaridade dos dados amostrais (não existência de efeito proporcional) pode ser obtida através do gráfico de média versus desvio-padrão, por linha e coluna, nas Figuras 4 e 5, nas faces A e B. Verifica-se que, na face A, as medidas de posição menores corresponderam às de dispersão mais elevadas no sentido da largura da peça (coluna), ainda que em poucos pontos; as medidas de dispersão mais elevadas no sentido da largura da peça (coluna), enquanto uma normalidade no sentido do comprimento (linhas) foi observada, conforme discutido anteriormente no gráfico "post plot" nas Figuras 2 e 3. Na face B da peça de madeira, as medidas de posição apresentaram comportamento semelhante na dispersão para valores baixos e altos determinados para a dureza, indicando que menores valores foram encontrados nessa face da peça.

A maior variabilidade das medidas na face B diz respeito à maior heterogeneidade da madeira em relação à posição medula-casca nessa face, ou seja, os pontos amostrados pertencem a mais de um anel anual de crescimento da face tangencial da peça de madeira amostrada.

Os resultados na análise geoestatística estão apresentados no Quadro 2, indicando dependência espacial da dureza nas duas faces da peça. Modelos isotrópicos e anisotrópicos foram considerados e avaliados pela validação cruzada. Com base nesse critério, decidiu-se pelo modelo isotrópico, ou seja, o padrão da estrutura espacial é o mesmo em todas as direções; se por um lado, detalha menos a estrutura espacial de correlações, por outro é mais simples. Os semivariogramas experimentais foram construídos considerando-se as distâncias (h) nas direções $0^{\circ}, 45^{\circ}, 90^{\circ}$ e $135^{\circ}$. O modelo exponencial, [y(h)] $=\mathrm{C}_{\mathrm{o}}+\mathrm{C}\left(1-\mathrm{e}^{(-3 \mathrm{~h} / \mathrm{a})}\right)$ para $0<\mathrm{h}<\mathrm{d}$, em que dé a máxima distância que o semivariograma é definido, foi o que melhor ajustou os dados de dureza nas faces A e B. 

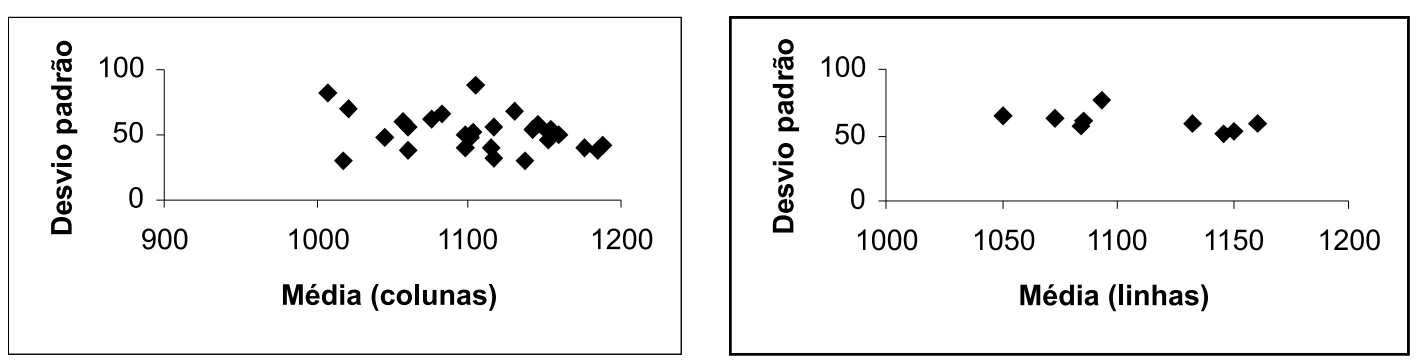

Figura 4 - Gráficos de dispersão das médias versus desvio-padrão da variável dureza $\left(\mathrm{kgf} / \mathrm{cm}^{2}\right)$ amostradas nos sentidos das colunas e linhas na face A.

Figure 4 -Dispersion graph of means versus standard deviation of variable hardness $\left(\mathrm{kgf} / \mathrm{cm}^{2}\right)$ sampled in the direction of columns and lines in side A.
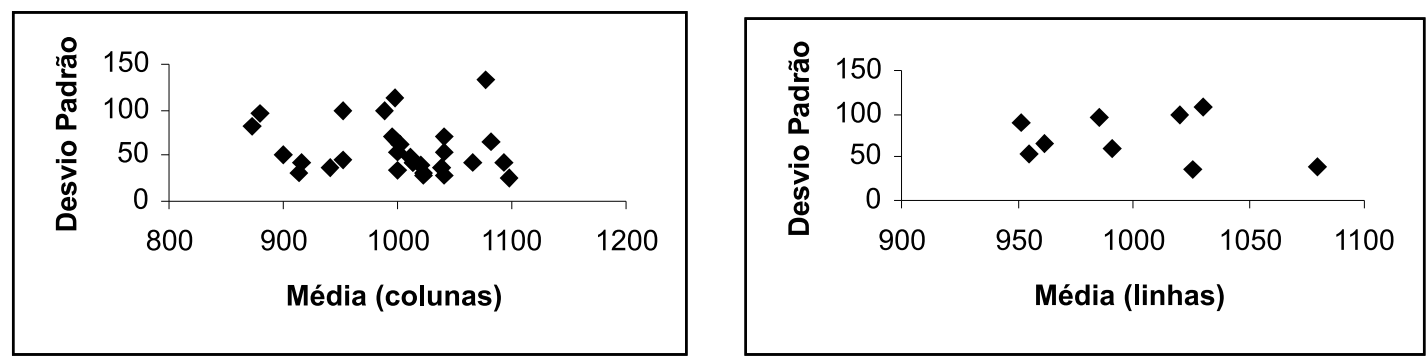

Figura 5 - Gráficos de dispersão das médias versus desvio-padrão da variável dureza $\left(\mathrm{kgf} / \mathrm{cm}^{2}\right)$ amostradas nos sentidos das colunas e linhas na face B.

Figure 5 - Dispersion graph of means versus standard deviation of variable hardness $\left(\mathrm{kg} / \mathrm{cm}^{2}\right)$ sampled in the direction of columns and lines in side $B$.

Quadro 2 - Parâmetros variográficos da dureza da madeira nas faces A e B

Table 2 - Variographic parameters of hardness in sides A and B

\begin{tabular}{ccccccc}
\hline Face & $\begin{array}{c}\text { Modelos } \\
\text { Ajustados }\end{array}$ & $\mathrm{C}_{\mathrm{o}}$ & $\mathrm{C}_{\mathrm{o}}+\mathrm{C}_{1}$ & $\mathrm{a}(\mathrm{mm})$ & $\mathrm{C}_{1} / \mathrm{C}_{\mathrm{o}}+\mathrm{C}_{1}$ & $\mathrm{R}^{2}$ \\
\hline A & EXP & 90 & 5514,0 & 223,8 & 0,984 & 0,971 \\
$\mathrm{~B}$ & EXP & 480 & 8070,0 & 149,7 & 0,941 & 0,981 \\
\hline
\end{tabular}

$\mathrm{C}_{\mathrm{o}}=$ efeito pepita, $\mathrm{C}_{\mathrm{o}}+\mathrm{C}_{1}=$ patamar, $\mathrm{a}=$ alcance, $\mathrm{C}_{1} / \mathrm{C}_{\mathrm{o}}+\mathrm{C}_{1}=$ grau dè dependência espacial e $\mathrm{R}^{2}$ = coeficiente de determinação.

Na Figura 6, apresentam-se os ajustes teóricos dos semivarigramas. Nesses ajustes, é possível observar que, em ambos os lados, a variável apresentou dependência espacial. O alcance (a) estabelece o limite de dependência espacial entre os pontos de leitura da dureza, isto é, nas distâncias iguais ou menores que o alcance, entende-se que os valores vizinhos da dureza estão espacialmente correlacionados e podem ser utilizados para se estimar valores em qualquer ponto entre eles. $\mathrm{O}$ alcance obtido na face $\mathrm{A}$ foi maior que na face $B$, sendo de $223,8 \mathrm{~mm}$ e de $149,7 \mathrm{~mm}$, respectivamente, indicando maior continuidade na distribuição da variável dureza na face A. Nesse caso, as amostras devem ser tiradas na peça a uma distância maior que o alcance, sendo, portanto, independentes. $\mathrm{O}$ "efeito pepita" $\left(\mathrm{C}_{0}\right)$ refere-se ao valor do semivariograma na interceptação do eixo Y e representa o comportamento da variação ao acaso. Nesse caso, foram considerados os semivariogramas com forte dependência espacial, sendo a contribuição $\left(C_{1}\right)=0,75$ do patamar nas faces A e B, de 0,984 e 0,941, respectivamente.

Com base nos ajustes dos semivariogramas, foi possível a construção de mapas de superfície por "krigagem" ordinária para a variável dureza em cada face, caracterizando o comportamento da dureza na peça de paraju, conforme se pode observar nas Figuras 7 e 8 .

R. Árvore, Viçosa-MG, v.30, n.4, p.651-657, 2006 

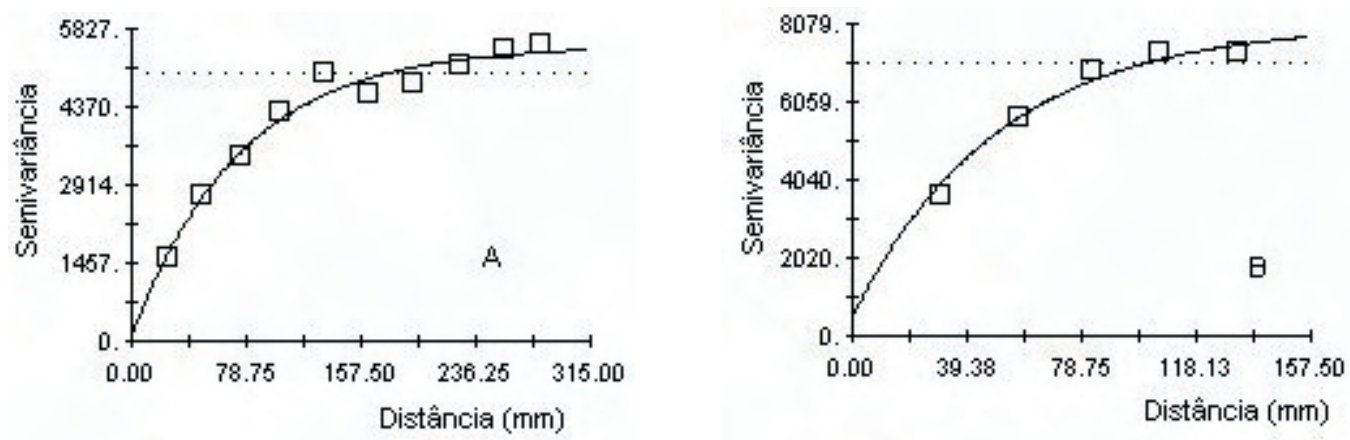

Figura 6 - Semivariograma experimental e teórico da dureza nas faces A e B.

Figure 6-Experimental and theoretical semivariograms of hardness in sides $A$ and $B$.
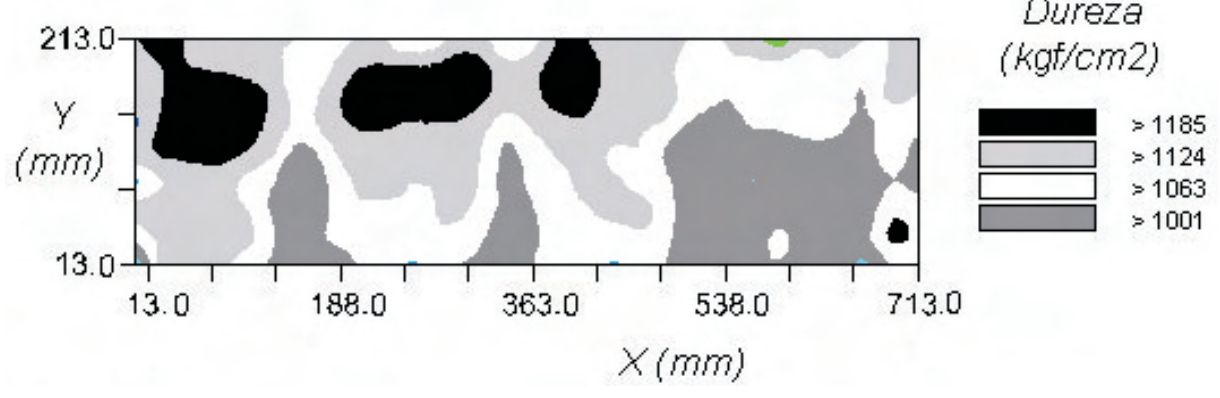

Figura 7 - Interpolação por krigagem da dureza na face A.

Figure 7 - Krigage interpolation of hardness in side $A$.

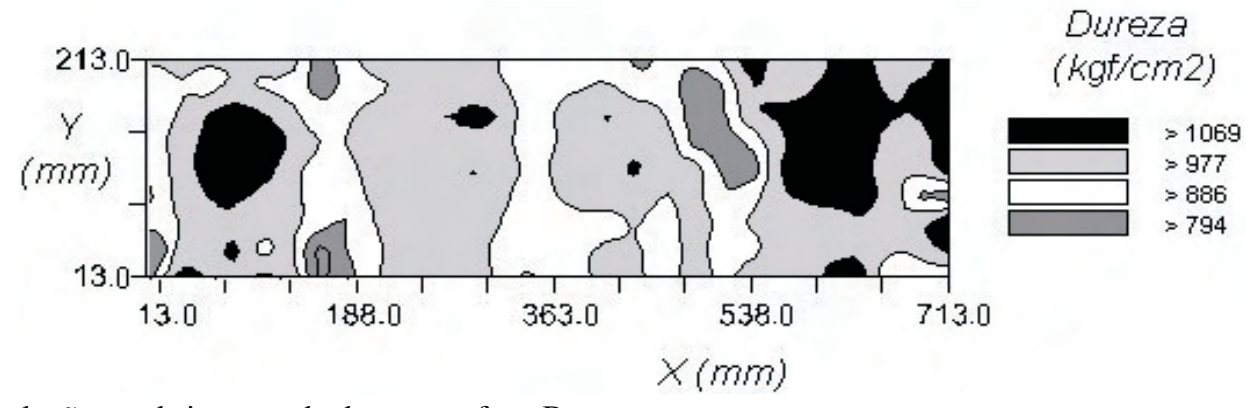

Figura 8 - Interpolação por krigagem da dureza na face B.

Figure 8 - Krigage interpolation of hardness in side B.

Os mapas diferem um pouco quanto à forma, entretanto percebe-se que a dureza, nas faces A e B, apresenta sub-regiões de concentração, como discutido anteriormente nos gráficos "post plot". A dureza apresentou maiores valores na face A em relação à face $\mathrm{B}$.

R. Árvore, Viçosa-MG, v.30, n.4, p.651-657, 2006

\section{CONCLUSÃO}

- A metodologia baseada na estatística espacial, ou geoestatística, mostrou-se viável na caracterização da propriedade em estudo, indicando uma estrutura com forte grau de dependência espacial entre os valores. 
- A dureza da madeira na face A em relação à face B apresentou menor coeficiente de variação, maior valor médio e maior continuidade, sendo, portanto, mais homogênea.

- Aplicação prática dessa técnica no fornecimento de subsídios para a determinação da distância ótima entre amostras (alcance) em peças de madeira, contribuindo para a tomada de decisão na área de tecnologia da madeira no estudo dessa propriedade mecânica.

- Há a necessidade de outros estudos da técnica aplicados às faces radiais de peças de madeiras, por estas possuírem elevada variabilidade de propriedades e por representarem toda a variação da madeira formada na direção medula-casca.

\section{REFERÊNCIAS BIBLIOGRÁFICAS}

AlZATE, S. B. A. Caracterização da madeira de árvores de clones de Eucalyptus grandis, E. saligna e E. grandis $x$ urophylla. 2004. 133f. Tese (Doutorado em Recursos Florestais) - Escola Superior de Agricultura “Luiz de Queiroz”, Piracicaba, 2004.

ASSOCIAÇÃO BRASILEIRA DE NORMAS TÉCNICAS - ABNT. Ensaios físicos e mecânicos da madeira - MB-26/40. Rio de Janeiro: 1940. 16p.

ISAAKS, E.H.; SRIVASTAVA, R.M. An introduction to applied geoestatistics, New York: Oxford University Press, 1989. 561p.

KRIGE, D. G. A statistical approach to some basic mine evaluation problems on the Witwatersrand. Journal South African. Institute. Minning Metttal, n. 52, p. 119-139, 1951.

OLIVEIRA, J. T. S. Caracterização da madeira de eucalipto para a construção civil. 1997. 429f. Tese (Doutorado em Engenharia Civil) - Escola Politécnica, Universidade de São Paulo, São Paulo, 1997.

PIMENTEL GOMES, F. Estatística moderna na agropecuária. 3. ed. Piracicaba: Patafos, 1987.

RIBEIRO JÚNIOR, P. J. Métodos geoestatísticos no estudo da variabilidade espacial de parâmetros do solo. 1995. 99f. Dissertação (Mestrado em Agronomia). Escola Superior de Agricultura Luiz de Queiroz, Piracicaba, 1995.
ROBERTSON, G. P. Geoestatistics in ecology: interpolating with known variance. Ecology, v. 68, p. 744-748, 1987.

ROBERTSON, G. P. GS+: Geoestatistics for the environmental sciencies - GS+ User's Guide. Plainwell, Gamma Desing Software, 1998. 152p.

SILVA, J. C. Caracterização da madeira de $E$. grandis de diferentes idades, visando a sua utilização na indústria moveleira. 2002. 160f. Tese (Doutorado em Ciências Florestais) - Universidade Federal do Paraná, Curitiba, 2002.

SOUZA, Z. M. et al. Variabilidade espacial de atributos físicos de um latossolo vermelho sob cultivo de cana-de-açucar. Revista Brasileira de Engenharia Agrícola e Ambiental, v. 8, n. 1, p. 51-58, 2004.

USHIZIMA, T. M.; BERNARDI, J. V. E.; LANDIM, P. M. B. Estudo da distribuição espacial do angico (Anadenanthera peregrina) na floresta estadual "Edmundo Navarro de Andrade" - Rio Claro, Sp, Brasil, empregando metodologia geoestatística. HOLOS Environment, v. 3, n. 1, p. 59-73, 2003.

VIEIRA, S.R.; DECHEN, S.C.F.; MARIA, I.C. Uso de geoestatística no mapeamento de atributos de solo e planta. In: CONGRESSO E FEIRA PARA USUÁRIOS DE GEOPROCESSAMENTO DAAMERICA LATINAGIS BRASIL 98, 4., 1998, Curitiba. Anais... Curitiba: 1998. CD-ROM.

VIEIRA, S. R. Geoestatistica em estudos de variabilidade espacial do solo. In:Tópicos em Ciência do Solo I. Viçosa, MG. : Sociedade Brasileira de Ciência do Solo. 2000. 352p.

WARRICK , A. W.; NIELSEN, D. R. Spatial variability of soil physical properties in the field. In: HILLEL, D. (Ed.). Applications of soil physics. New York: Academic Press, 1980. p. 319-324.

WEBSTER, R.; OLIVER, M.A. Statistical methods in soil and land resource survey. Oxford: Oxford University Press, 1980. 316p.

R. Árvore, Viçosa-MG, v.30, n.4, p.651-657, 2006 